\title{
The effects of focus on scope relations between quantifiers and negation in Korean
}

\author{
Keunhyung Park \& Stanley Dubinsky*
}

\begin{abstract}
This paper addresses the effects of focus-marking (i.e. nun-marking) on the scope of quantified expressions in Korean negation constructions and shows how these inform the analysis of Korean negation constructions generally. Specifically, highlighting the "Rigid Scope" properties of Korean (in contrast with English), focus-marking in Korean negation constructions eliminates quantifier/negative scope ambiguities. In all cases but one, a focus-marked element has scope over all others. The anomalous case involving contrastive focus of object universal quantifiers brings the semantics of quantifiers into opposition with the semantics of contrastive focus.
\end{abstract}

Keywords. Korean; English; syntax; semantics; scope relation; quantifier raising; focus; contrastive focus; negation.

1. Scope ambiguity in English and rigidity in Korean. English and Korean quantified expressions display different scope possibilities when more than one such expression occurs in a single clause. In the English example (1), relative scope of existential and universal quantifiers is ambiguous (some >every or every>some) and two different readings are available. In contrast, Korean example (2) has the same two quantified expressions, and there is no ambiguity (the subject quantifier has "rigid" scope over the object. See Choi 1999, H-J Kim 2007). ${ }^{1}$

(1) Someone answered every question.

$\exists>\forall / \quad \forall>\exists$

$\begin{array}{ll}\text { nwukwunka-ka } & \text { motun mwuncey-lul } \\ \text { someone-NOM } & \text { every question-ACC } \\ \text { 'Someone answered every question.' }\end{array}$

phwul-ess-ta $\exists>\forall \mid * \forall>\exists$ answer-PST-DECL

This is accounted for by the hypothesis that Korean subjects need not move to Spec,TP, unlike English. Each of the quantified NPs in (1) can take scope over the other, resulting in scopal ambiguity. In the LF representation of the English example (1), (1'), someone and its intermediate trace c-command every question yielding $\exists>\forall$. At the same time, every question ccommands the lowest trace of someone yielding $\forall>\exists$ (see Aoun \& Li 1993). In contrast, Korean example (2) has a rigid scope interpretation (i.e. scope interpretation of the quantified expressions is restricted to their surface order). In the LF representation of this example, $\left(2^{\prime}\right)$, subject and object are generated in Spec,vP and Spec, VP, respectively, and QR results in each argument adjoining to its own vP or VP.

$$
\begin{aligned}
& \text { LF: [тр Someone } 1 \text { [тр } \mathrm{t}^{\prime}{ }_{1} \text { [vр every question }{ }_{2} \text { [vр } \mathrm{t}_{1} \text { answered } \mathrm{t}_{2} \text { ]]]] } \\
& \text { LF: [vP1 nwukwunka-ka1 [vP1 } \mathrm{t}_{1} \text { [vP2 motun mwuncey-lul } 2 \text { [vP2 } \mathrm{t}_{2} \text { phwul-ess-ta]]] }
\end{aligned}
$$

\footnotetext{
* Authors: Keunhyung Park, University of South Carolina (kp4@email.sc.edu) \& Stanley Dubinsky, University of South Carolina (dubinsky@sc.edu).

${ }^{1}$ Chinese also shows scope relations similar to Korean when two quantified expressions are in a simple sentence. Huang (1982) following the Isomorphic Principle insists that the c-command relation at SS is maintained at LF (see Huang (1982) and Lee (1986) for Chinese quantification).
} 
2. Scope of NP quantifiers relative to negation. As described in Section 1, the relative scope of quantified expressions in sentences having more than one of them is determined differently in English and in Korean. In this section, we examine the interpretation of quantified subjects and objects, respectively, in negated clauses. It will be shown that Korean quantifiers are more likely to have ambiguous scope relative to negation, and that this ambiguity is independent of whether the quantified expression is a subject or an object (as we might expect in a language which does not require movement of subjects to a higher functional projection).

2.1. SCOPE RELATIONS IN ENGLiSh. Relative scope of quantified NPs and negation in English is determined in part by the position of the quantified NP and in part by the nature of the quantifier. With a quantified subject NP, scope is ambiguous with a universally quantified NP, as in (3a), but inverse scope is disallowed with an existentially quantified NP, as in (3b). In contrast, with a quantified object NP, scope relations are quite different. Example (4a) is unambiguous and does not allow the object to scope over negation. Example (4b) is ambiguous and example (4c), with NPI any, only has an interpretation that is the opposite of (4a) (see Beghelli \& Stowell 1997).
a. Every student didn't answer the question.
b. One student didn't answer the question.$$
\text { a. Hana didn't answer every question. }
$$$$
\text { b. Hana didn't answer one question. }
$$$$
\text { c. Hana didn't answer any question. }
$$

$$
\begin{gathered}
\forall>\text { NEG / NEG }>\forall \\
\exists>\text { NEG / *NEG }>\exists \\
* \forall>N E G / N E G>\forall \\
\exists>\text { NEG / NEG }>\exists \\
\forall>N E G / * N E G>\forall
\end{gathered}
$$

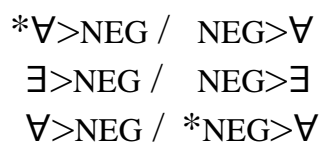

Example (3a) is ambiguous between the meanings 'There is no student such that said student answered the question' and 'It is not the case, for every student, that each one answered the question (i.e. some student didn't)'. In contrast, (3b) can only mean 'There is one student such that said student did not answer the question'. When negation is higher in the structure (e.g. when the object NP is quantified), we find that scope relations between the quantified NP and negation are quite different. (4a) only means 'It is not the case, for every question, that Hana answered it (i.e. there is at least one question that she did not answer).' Example (4b), with an indefinite existential quantifier, is ambiguous and means either 'There is one question such that Hana did not answer it' or 'It is not the case that Hana answered (even) one question.' Example (4c), with NPI any, only has an interpretation that is the opposite of (4a), and means 'For every question, it is not the case that Hana answered it.'

2.2. SCOPE RELATIONS IN KOREAN. Korean has two different types of negation, short-form (SFN) and long-form (LFN), and there is substantial disagreement regarding wide-scope readings of negation in SFN constructions (see Hagstrom 2000, Han et al. 2007, Kim 2000). For this reason, we confine our examination of the Korean facts to those involving LFN. In Korean LFN, quantifier/negative scope is ambiguous, and this is due to the possibility of there being either V-raising or V-lowering in the structure (see below). Accordingly, while preferred interpretations correspond to constituents' surface order, the quantified NP, with sufficient context, can also be interpreted as having scope below negation. 
motun haksayng-i

every student-NOM ku mwuncey-lul the question-ACC phwul-ci
answer-NMLZ

phwul-ci answer-NMLZ

phwul-ci

answer-NMLZ

phwul-ci answer-NMLZ an-ha-ss-ta

NEG-do-PST-DECL $\forall>\mathrm{NEG} / \mathrm{NEG}>\forall$

an-ha-ss-ta

NEG-do-PST-DECL $\forall>$ NEG / NEG $>\forall$

an-ha-ss-ta

NEG-do-PST-DECL $\exists>\mathrm{NEG} / \mathrm{NEG}>\exists$

an-ha-ss-ta

NEG-do-PST-DECL

While this ambiguity appears to violate the Rigid Scope restriction in Korean, wherein scope interpretations are decided by the linear order of quantified elements at PF, we know (based on an analysis proposed in Han et al. 2007) that the entire verbal complex phwul-ci-an-ha-ss-ta can either raise to the head of CP or lower to the head of VP. Thus, since the head of NegP is included in this complex, negation can be either outside or inside the scope of the quantified subject or object nominals.

3. nun-marked predicates and the scope of negation. This section explores the effects of focus marking on the predicate in Korean negative sentences, examining the behavior of focus marking on the nominalized verb in LFN constructions. As we shall see presently, focus marking disrupts the usual scope interpretations available for these and negation. When the nominalized verb is focus-marked, negation must have scope over all SUBJ and OBJ quantifiers.

LFN constructions, significantly contrasting with SFN constructions, have a unique structure of nominalization with a special nominalizer $c i$. With this marker, the main verb is nominalized, and negation is attached to the inserted $h a$ auxiliary verb generating a negative auxiliary complex at the end of sentence. This nominalized verb phwul-ci 'answer-ci' can also be focus-marked like other subject and object nominals, as in (9)-(12). Here, the nominalized verb (e.g. phwul-ci 'answer-ci') together with its associated negative complex (e.g. an-ha-ss-ta 'didn't') functions as a unit constituent (see Kim \& Park 2000 for evidence). The focus-marked ci phrase (i.e. V-ci-nun) must move to the head of FocP, which is higher than vP/VP-adjoined quantifiers. Thus, the focus-marked V-ci-NEG-AuxV complex has wider scope than other NP quantifiers.

$$
\begin{array}{lll}
\begin{array}{l}
\text { motun haksayng-i } \\
\text { every student-NOM }
\end{array} & \begin{array}{l}
\text { ku mwuncey-lul } \\
\text { the question-ACC }
\end{array} & \begin{array}{l}
\text { phwul-ci-nun } \\
\text { answer-NMLZ-FOC }
\end{array} \\
\begin{array}{lll}
\text { Hana-ka } & \begin{array}{l}
\text { motun mwuncey-lul } \\
\text { Hana-NOM }
\end{array} & \begin{array}{l}
\text { phwul-ci-nun } \\
\text { every question-ACC }
\end{array}
\end{array}
\end{array}
$$

an-ha-ss-ta

NEG-do-PST-DECL

$* \forall>$ NEG / NEG $>\forall$

an-ha-ss-ta

NEG-do-PST-DECL

$* \forall>$ NEG / NEG $>\forall$ 
(11)

han haksayng-i
one student-NOM

ku mwuncey-lul

the question-ACC

(12) phwul-ci-nun

answer-NMLZ-FOC

phwul-ci-nun

answer-NMLZ-FOC an-ha-ss-ta

NEG-do-PST-DECL

*ヨ>NEG / NEG>

an-ha-ss-ta

NEG-do-PST-DECL

*ヨ>NEG / NEG>

Focus-marked nominalized verbs (e.g. phwul-ci-nun) must undergo head-raising only, thus disambiguating the two possible scope interpretations. This is a result of the affix nun having a [+FOCUS] feature which must be checked by LF. Accordingly, phwul-ci-nun 'answer-ci-nun' in examples (9)-(12) must undergo head movement from $\mathrm{VP}^{0}$ through $\mathrm{T}^{0}$ and $\mathrm{C}^{0}$ to $\mathrm{Foc}^{0}$. This operation is shown here in $\left(9^{\prime}\right)$. In $\left(9^{\prime}\right)$, the verb phwul 'answer' is nominalized with $c i$ and focusmarked with nun, and then moves to an 'not' and then to hass 'did'. The focus feature on nun forces it to move to $t a$ (head of CP) and then further to the head of FocP, where its [+FOCUS] feature is checked. Notice that this movement operation places the V-ci-FOCUS-NEG-AuxV-Tense complex phwul-ci-nun-an-ha-ss-ta wholly outside of TP, such that negation here has scope over any quantified NP, inclusive of the subject.

\section{$\left(9^{\prime}\right) \quad$ Before head-movement}

[FocP [motun haksayng-i [NegP[V-ciP ku mwuncey-lul phwul-ci-nun] an] hass-ta] FOC]

After head-movement (Raising)

[FocP [motun haksayng-i [NegP[V-ciP ku mwuncey-lul $\left.t_{1}\right]_{t_{2}} \mathrm{t}_{3}-\mathrm{t}_{4}$ ] phwul-ci-nun -an $_{2}$-hass 3 - $\left.\mathrm{ta}_{4}\right]$

Based on this analysis, quantifier scope data shown in (9)-(12) are expected. Quantified nominal expressions (e.g. both subject and object) are assumed to adjoin to TP (or vP) at LF. For its part, the focus-marked $c i$ phrase (i.e. V-ci-nun) must move to the head of FocP, which is higher than the TP-adjoined quantified nominals. Thus, the focus-marked V-ci-NEG-AuxV complex has wider scope than other quantified NPs, as we have seen.

4. nun-marked NP quantifiers and the scope of negation. This section explores the effects of focus marking directly on quantified expressions in Korean negative sentences. Recall first, the fact that LFN constructions containing quantified subjects and objects are uniformly ambiguous with respect to the relative scope of the quantifier and negation, as seen above in (5)-(8). Previously, we appealed to Han et al. 2007 for an explanation of the ambiguity in the attested sentences, relying on the possibility of optional head raising or lowering of the verbal complex.

When the quantified nominals in the sentences below are marked with $-(n) u n$, in much the same way that focus marking on the verbal complex reduces the possibilities for ambiguity in Section 3, we predict (and do find) that focus marking on quantified nominals eliminates otherwise-available interpretations. However, as we see here, the behavior of focus-marked quantified expressions is not entirely predictable and is confounded by the nature of the quantifiers and some ambiguities in the interpretation of the "focus" marker $-(n)$ un. We find that nun-marked NP quantifiers typically take obligatory scope over negation (as might be expected if they move to check a [FOCUS] feature in Spec,FocP), except in the case of contrastively focused universal quantifiers in object position. We see this to be the case in examples (13) (16), where (14) anomalously disallows the focus-marked universally quantified object motun mwuncey 'every question' having scope over NEG. 
Hana-ka motun haksayng-un ku mwuncey-lul

every student-FOC

the question-ACC Hana-NOM every question-FOC

han haksayng-un one student-FOC

ku mwuncey-lul the question-ACC Hana-NOM

\author{
han mwuncey-nun \\ one question-FOC
}

phwul-ci answer-NMLZ

phwul-ci answer-NMLZ

an-ha-ss-ta

NEG-do-PST-DECL $\forall>\mathrm{NEG} ; *$ NEG $>\forall$

an-ha-ss-ta NEG-do-PST-DECL $* \forall>$ NEG / NEG $>\forall$

an-ha-ss-ta NEG-do-PST-DECL $\exists>\mathrm{NEG} ; *$ NEG>ヨ

phwul-ci an-ha-ss-ta answer-NMLZ NEG-do-PST-DECL $\exists>\mathrm{NEG} / *_{\mathrm{NEG}}>\exists$

Given that the disappearance of ambiguity arises from the insertion of nun-marking, rather than from any other properties of these expressions, we will seek to explain this change of interpretations in terms of the interactions between quantification and focus. Note that a nunmarked existentially quantified object, as in (16), takes obligatory scope over negation. In contrast, we see that negation has obligatory scope over a nun-marked universally quantified object in (14). Section 5 will take up the problems inherent in focus interpretations of universally quantified objects and propose an analysis of these which explains their anomalous interpretations with respect to negation by appeal to a previously unnoted ability of object nunmarking to function as a contrastive focus-marker of its entire containing predicate.

5. nun-marked universal quantifiers and negation. This section takes up restricted scope options shown in (14). Example (14) appears to challenge our assertion that a [FOCUS] feature need be checked in Spec,FocP, if in fact FocP is above NegP. For (14), we are faced with the following facts: (i) 'every question' has focus marking, (ii) focus-marked elements need to have [FOCUS] checked in Spec,FocP, and (iii) FocP has scope over NegP. We would therefore expect either that 'every question' should have scope over negation or that the sentence should be ungrammatical/uninterpretable. What we find, however, is that (14) does have a plausible interpretation, but that interpretation involves contrastive focus of the entire VP, which itself contains the lowered head of NegP, thereby allowing both checking of [FOCUS] and NEG $>\forall$ scope. The ability of a nun-marked object to signal contrastive focus of its containing VP has not previously been noticed and is illustrated in examples (17) and (18), the first of which is ambiguous. Note that these examples do not involve negation at all.
Hana-ka
han mwuncey-nun
phwul-ess-ta
Hana-NOM one question-FOC
answer-PST-DECL

Object Q-focus: ' $\mathrm{H}$ answered one Q, (in contrast with other Qs).'

Presupposition: there is at least one more question in addition to the one $\mathrm{H}$ answered.

Predicate focus: ' $\mathrm{H}$ answered one $\mathrm{Q}$, (in contrast to ignoring it).'

Presupposition: there is at least one other thing $\mathrm{H}$ could have done besides answering. 


$$
\begin{array}{lll}
\text { Hana-ka } & \text { motun mwuncey-nun } & \text { phwul-ess-ta } \\
\text { Hana-NOM } & \text { every question-FOC } & \text { answer-PST-DECL }
\end{array}
$$

Object Q-focus: \#'H answered every Q, (in contrast with other Qs).'

\#Presupposition: there is at least one more question in addition to every $\mathrm{Q} H$ answered.

Predicate focus: ' $\mathrm{H}$ answered every $\mathrm{Q}$, (in contrast to ignoring them).'

Presupposition: there is at least one other thing $\mathrm{H}$ could have done besides answering.

Example (17) is ambiguous, with the nun-marked object signaling contrast with other potential objects (e.g. other questions) or contrast with other potential predicates (e.g. other actions besides answering). While there is nothing underivable about an Object Q-focus reading for (18), the interpretation is anomalous, since every is exhaustive with respect to the set of questions (i.e., contrasting every question with the empty set makes little sense). Of course, if the noun itself were contrasted, then object focus would be possible.

The derivation shown in (17') illustrates how Object Q-focus for (17) is derived, with han mwuncey-nun moving to Spec,FocP. In (17"), the Predicate focus reading is derived through adjunction of han mwuncey-nun to VP, with the entire VP moving to Spec,FocP to check the [FOCUS] feature.

\section{$\left(17^{\prime}\right) \quad$ Object Q-focus}

PF: [FocP [CP Hana-ka [vP [NP han mwuncey-nun] phwul-ess-ta]]]

LF: [FocP [NP han mwuncey-nun $]_{1}\left[\mathrm{CP}\right.$ Hana-ka [vP $\quad \mathrm{t}_{1} \quad$ phwul-ess-ta] $]$

\section{$\left(17^{\prime \prime}\right)$ Predicate focus}

PF: [FocP

[CP Hana-ka [vP [NP han mwuncey-nun] phwul-ess-ta]]]

LF: $\left[\right.$ FocP $\left[\mathbf{v P}[\mathrm{NP} h a n \text { mwuncey-nun }]_{1}\left[\mathbf{v P} \quad \mathrm{t}_{1} \quad \text { phwul-ess-ta] }\right]_{2}\left[\mathrm{CP}\right.\right.$ Hana-ka $\left.\mathrm{t}_{2} \quad\right]$

In contrast to (17), example (18) only has a Predicate focus interpretation. The derivation shown in (18') illustrates how Object Q-focus for (18) would be derived, were it not anomalous, with motun mwuncey-nun moving to Spec,FocP. In (18"), the Predicate focus reading is derived through adjunction of motun mwuncey-nun to VP, with the entire VP moving to Spec,FocP.

\section{(18') Object $\mathbf{Q}$-focus}

PF: [FocP [CP Hana-ka [vP [NP motun mwuncey-nun] phwul-ess-ta]]]

LF: \#[FocP [NP motun mwuncey-nun] $]_{1}$ [CP Hana-ka [vP $\quad t_{1} \quad$ phwul-ess-ta]]]

\section{$\left(18^{\prime \prime}\right)$ Predicate focus}

PF: [FocP [CP Hana-ka [vP [NP motun mwuncey-nun] phwul-ess-ta]]]

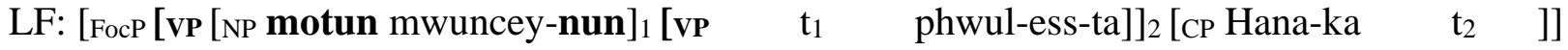

Returning now to the anomalous data shown in (14) and (16), we can explain how it is that a focused universally quantified object cannot take scope over negation. Example (14) is derived in an identical manner to (18), except that negation is involved. In (14'), the focus-marked universally quantified object motun mwuncey-nun 'every question-nun' cannot have contrastive focus and therefore cannot move to Spec,FocP where it would check [FOCUS] and take scope over NEG. In (14"), motun mwuncey-nun adjoins to VP, where the [FOCUS] is visible at the left edge of this phrase for checking. This allows the derivation of the Predicate focus reading with the entire VP moving to Spec,FocP. In this derivation, though, NEG continues to M-command (and maintain scope over) the object universal quantifier that is adjoined to VP, leaving NEG $>\forall$. 


\section{(14') Object Q-focus}

PF: [FocP [CP Hana-ka [vP [NP motun mwuncey-nun] phwul-ci-an-hass-ta]]]

LF: \#[FocP [NP motun mwuncey-nun $]_{1}$ [CP Hana-ka [vP $\quad t_{1}$ phwul-ci-an-hass-ta]]]

\section{$\left(14^{\prime \prime}\right)$ Predicate focus}

PF: [FocP [CP Hana-ka [vP [NP motun mwuncey-nun] phwul-ci-an-hass-ta]]]

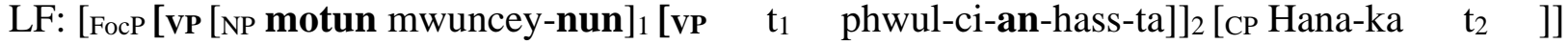

6. Conclusion. Adopting a synthesis of the analyses proposed in Han et. al 2007 and Kim \& Park 2000, we suggested that scope ambiguity in Korean negative constructions results from the possibility of both head-raising and head-lowering of the verb and its functional complex. In the present study, we showed that nun-marking of quantified arguments or of a nominalized verb eliminates the usual quantifier/negative scope ambiguities. In all cases but one, this disambiguation was seen to be the result of movement of nun-marked elements at LF to a FocP projection outside of CP. However, contrastive focus of object universal quantifiers is found to be impossible, where [FOCUS] on such objects typically serves to contrastively focus the entire containing VP.

\section{References}

Aoun, Joseph \& Audrey Yen-Hui Li. 1993. Syntax of scope. Cambridge. MA: MIT Press. https://mitpress.mit.edu/books/syntax-scope.

Beghelli, Filippo \& Tim Stowell. 1997. Distributivity and negation: The syntax of each and every. In Anna Szabolcsi (ed.), Ways of scope taking. Studies in Linguistics and Philosophy 65.71-107. Dordrecht: Springer. https://link.springer.com/chapter/10.1007/978-94-011-5814-5_3.

Choi, Young-Sik. 1999. Negation, its scope and NPI licensing in Korean. In Rebecca Daly \& Anastacia Riehl (eds.), Proceedings of Eastern States Conference on Linguistics `99 (ESCOL). 25-36. Ithaca, NY: CLC Publications.

Hagstrom, Paul. 2000. Phrasal movement in Korean negation. In Proceedings from SCIL 9: The 9th Student Conference in Linguistics. 127-142. Cambridge, MA: MITWPL. https://www.bu.edu/linguistics/UG/hagstrom/papers/SCIL9.pdf.

Han, Chung-hye, Jeffrey Lidz \& Julien Musolino. 2007. V-raising and grammar competition in Korean: Evidence from negation and quantifier scope. Linguistic Inquiry 38(1). 1-47. https://doi.org/10.1162/ling.2007.38.1.1.

Kim, Hyun-Ju. 2007. Acquisition of scope interaction of universal quantifiers and negation in Korean-English bilingual children. Unpublished ms. Stony Brook University.

Kim, Jong-Bok. 2000. The grammar of negation: A constraint-based approach. Stanford: CSLI Publications. https://web.stanford.edu/group/cslipublications/cslipublications/site/1575862301.shtml.

Kim, Jong-Bok \& Byung-Soo Park. 2000. The structure of long form negation and argument structure. Language Research 36(4). 715-733. 